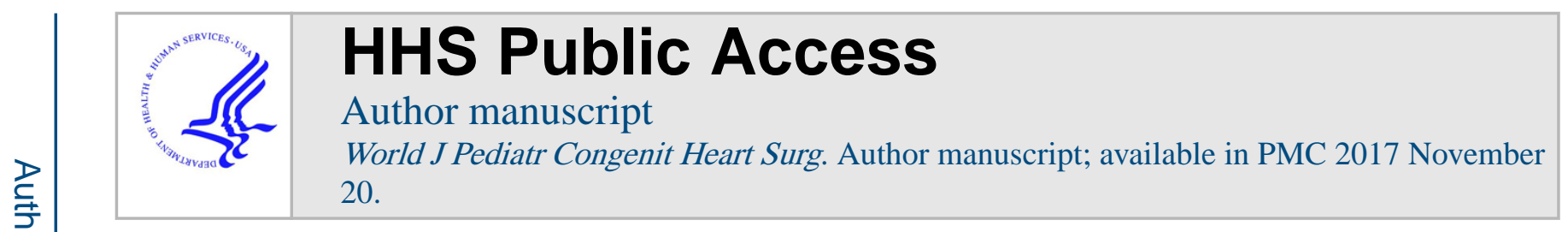

Published in final edited form as:

World J Pediatr Congenit Heart Surg. 2017 May ; 8(3): 391-393. doi:10.1177/2150135117692777.

\title{
Virtual Surgery for Conduit Reconstruction of the Right Ventricular Outflow Tract
}

Chin Siang Ong, MBBS ${ }^{1}$, Yue-Hin Loke, MD², Justin Opfermann, $\mathbf{M S}^{2}$, Laura Olivieri, MD², Luca Vricella, MD ${ }^{1}$, Axel Krieger, PhD $^{3}$, and Narutoshi Hibino, MD, PhD ${ }^{1}$

${ }^{1}$ Division of Cardiac Surgery, Johns Hopkins Hospital, Baltimore, MD, USA

2Division of Cardiology, Children's National Health System, Washington DC, USA

${ }^{3}$ Sheikh Zayed Institute for Pediatric Surgical Innovation, Children's National Health System, Washington DC, USA

\section{Abstract}

Purpose-Virtual surgery involves the planning and simulation of surgical reconstruction using three-dimensional (3D) modeling based upon individual patient data, augmented by simulation of planned surgical alterations including implantation of devices or grafts. Here we describe a case in which virtual cardiac surgery aided us in determining the optimal conduit size to use for the reconstruction of the right ventricular outflow tract.

Description-The patient is a young adolescent male with a history of tetralogy of Fallot with pulmonary atresia, requiring right ventricle-to-pulmonary artery (RV-PA) conduit replacement. Utilizing preoperative magnetic resonance imaging data, virtual surgery was undertaken to construct his heart in 3D and to simulate the implantation of three different sizes of RV-PA conduit $(18,20$, and $22 \mathrm{~mm})$.

Evaluation-Virtual cardiac surgery allowed us to predict the ability to implant a conduit of a size that would likely remain adequate in the face of continued somatic growth and also allow for the possibility of transcatheter pulmonary valve implantation at some time in the future.

Subsequently, the patient underwent uneventful conduit change surgery with implantation of a 22$\mathrm{mm}$ Hancock valved conduit. As predicted, the intra-thoracic space was sufficient to accommodate the relatively large conduit size without geometric distortion or sternal compression.

Conclusion-Virtual cardiac surgery gives surgeons the ability to simulate the implantation of prostheses of different sizes in relation to the dimensions of a specific patient's own heart and thoracic cavity in $3 \mathrm{D}$ prior to surgery. This can be very helpful in predicting optimal conduit size, determining appropriate timing of surgery, and patient education.

Reprints and permission: sagepub.com/journalsPermissions.nav

Corresponding Author: Narutoshi Hibino, Division of Cardiac Surgery, The Johns Hopkins Hospital, Suite 7107, Sheikh Zayed Tower, 1800 Orleans St, Baltimore, MD 21287, USA. nhibino1@jhmi.edu.

Declaration of Conflicting Interests

The author(s) declared no potential conflicts of interest with respect to the research, authorship, and/or publication of this article. 


\section{Keywords}

virtual surgery; right ventricle-to-pulmonary artery (RV-PA) conduit surgery; computer-aided design; 3D reconstruction

"Virtual surgery" involves using patient data, usually radiological images, to create patientspecific three-dimensional (3D) models, so that planned surgical procedures, such as the implantation of devices or grafts, can be carried out in silico, before the actual surgery. It has been used in several surgical specialties, notably orthognathic and craniofacial surgery, ${ }^{1}$ but application to cardiac surgery has primarily addressed planned implantation of mechanical circulatory support devices. For congenital heart disease, 3D reconstruction of intracardiac anatomy ${ }^{2}$ and $3 \mathrm{D}$ printing of physical models ${ }^{3}$ have been used for cardiac surgical planning, but the use of virtual cardiac surgery remains novel. Here we describe a case in which virtual cardiac surgery aided us in determining the optimal conduit size to use for the reconstruction of right ventricular outflow tract (RVOT).

The patient is a young adolescent male with a history of tetralogy of Fallot with pulmonary atresia. He was initially palliated with a left Blalock-Taussig shunt at two months of age and then a central shunt at six months. At the age of one year and four months, he underwent central pulmonary artery reconstruction and placement of an 8-mm pulmonary valve homograft as RV-PA conduit. Two months later (at the age of one year and six months), he underwent ventricular septal defect closure and placement of a 12-mm Hancock valved RVPA conduit. Over the ensuing years, he has experienced satisfactory somatic growth and has progressively developed conduit stenosis. He has been monitored closely with serial echocardiograms as well as periodic evaluation with cardiac magnetic resonance imaging (MRI) studies. The RVOT pressure gradient on echocardiography at the age of 12 years and 1 month was $94 \mathrm{~mm} \mathrm{Hg}$ (peak) and $58 \mathrm{~mm} \mathrm{Hg}$ (mean). At the age of 12 years and 8 months, the patient underwent a cardiac MRI scan, which showed a right ventricular end-diastolic volume index of $83.2 \mathrm{~mL} / \mathrm{m}^{2}$ and a regurgitant fraction of $7.4 \%$. Utilizing digital data from the MRI study, virtual surgery was undertaken to construct his heart in 3D and to simulate the implantation of three different sizes of RV-PA conduit (18, 20, and $22 \mathrm{~mm}$; Figures 1 and 2). The 3D data and virtual surgery images were presented and discussed at the pediatric joint cardiology and cardiac surgery preoperative conference. Although conduit replacement was clearly indicated at this time on the basis of the estimated RVOT gradient and right ventricular pressure, it was reassuring to be able to simulate the geometry of conduit implantation with several sizes of device and predict the ability to implant a conduit of a size that would likely remain adequate in the face of continued somatic growth (anticipated adolescent growth spurt) and also allow for the possibility of transcatheter pulmonary valve (TPV) implantation at some time in the future. A recent long-term follow-up study of TPV replacement found that smaller TPVs are associated with a higher mean Doppler RVOT gradient at discharge and a high RVOT mean gradient at discharge is associated with TPV reintervention. ${ }^{4}$ Thus, a conduit with a larger diameter was better. Subsequent to this exercise, the patient underwent uneventful conduit change surgery with implantation of a 22mm Hancock valved conduit at age 13 years and 2 months. As predicted by the virtual surgery simulation, the intrathoracic space was sufficient to accommodate the relatively 
large conduit size without geometric distortion or sternal compression. Recovery was uneventful and he was discharged seven days after surgery.

Virtual cardiac surgery gives surgeons the ability to simulate the implantation of prostheses of different sizes in relation to the dimensions of a specific patient's own heart in 3D prior to surgery (Figure 1). This can be very helpful in predicting optimal conduit size. As one of the modes of conduit failure is chest wall compression, it is important to ensure there is enough space in the thorax prior to implantation. Thus, the relation of the virtual simulated conduit to the chest wall (Figure 2) is important. While we sometimes print 3D heart models of complex congenital heart disease for surgical planning and education, in this case, the anatomy of the heart itself is not complex. The images of the virtual simulated conduit superimposed on the patient's existing MRIs (Figure 2) are more useful than actual 3D printing to estimate the relation of the virtual simulated conduit to the chest wall.

Virtual cardiac surgery may also be helpful in determining appropriate timing of surgery. An example would be the case of a child with a moderate gradient across a previously implanted RV-PA conduit in combination with some degree of conduit valve regurgitation. Surgery in such a case would be elective, with numerous factors contributing to the determination of optimal timing. In light of the development of TPV replacement, ${ }^{5}$ there now exists considerable impetus to reduce the number of surgical RVOT procedures that may be required over a patient's lifetime by extending the functional life of an implanted valved conduit through the use of transcatheter devices. At the present time, the size (diameter) of the surgically implanted conduit is one of the principal determinants of the possibility of subsequent implantation of devices such as TPVs. The ability to predict surgical implantation of a conduit that is sufficiently large to assure a high likelihood of the possibility of eventual deployment of a TPV or similar device may be helpful to clinicians in determining the timing of surgical RVOT procedures that are elective.

With respect to the actual case illustrated in this report, the 4-mm difference in diameter between 18- and 22-mm RV-PA conduits may be physically quite small (approximately equal to the thickness of two coins), but the ability to implant a 22-mm conduit instead of an 18-mm conduit could mean one less major open-heart surgery for a pediatric patient with congenital heart disease. "Virtual conduit implantation" can be done using the patient's existing preoperative imaging data and does not cause any additional discomfort or inconvenience to the patient. Finally, it can be used as an educational tool for patients and parents as well as for clinical and academic discussions at preoperative conferences.

\section{Acknowledgments}

\section{Funding}

The author(s) received the following financial support for the research, authorship, and/or publication of this article: This study was funded in part by NIH 1R21HD090671; investigator Narutoshi Hibino, MD, PhD.

\section{Abbreviations and Acronyms}


MRI magnetic resonance imaging

RV-PA right ventricle-to-pulmonary artery

RVOT right ventricular outflow tract

TPV transcatheter pulmonary valve

\section{References}

1. Swennen GR, Mollemans W, Schutyser F. Three-dimensional treatment planning of orthognathic surgery in the era of virtual imaging. J Oral Maxillofac Surg. 2009; 67(10):2080-2092. [PubMed: 19761902]

2. Dydynski PB, Kiper C, Kozik D, Keller BB, Austin E, Holland B. Three-dimensional reconstruction of intracardiac anatomy using CTA and surgical planning for double outlet right ventricle: early experience at a tertiary care congenital heart center. World J Pediatr Congenit Heart Surg. 2016; 7(4):467-474. [PubMed: 27358302]

3. Vodiskar J, Kutting M, Steinseifer U, Vazquez-Jimenez JF, Sonn-tag SJ. Using 3D physical modeling to plan surgical corrections of complex congenital heart defects. Thorac Cardiovasc Surg. 2017; 65(1):31-35. [PubMed: 27177266]

4. Cheatham JP, Hellenbrand WE, Zahn EM, et al. Clinical and hemodynamic outcomes up to 7 years after transcatheter pulmonary valve replacement in the US melody valve investigational device exemption trial. Circulation. 2015; 131(22):1960-1970. [PubMed: 25944758]

5. Bonhoeffer P, Boudjemline Y, Saliba Z, et al. Percutaneous replacement of pulmonary valve in a right-ventricle to pulmonary-artery prosthetic conduit with valve dysfunction. Lancet. 2000; 356(9239):1403-1405. [PubMed: 11052583] 


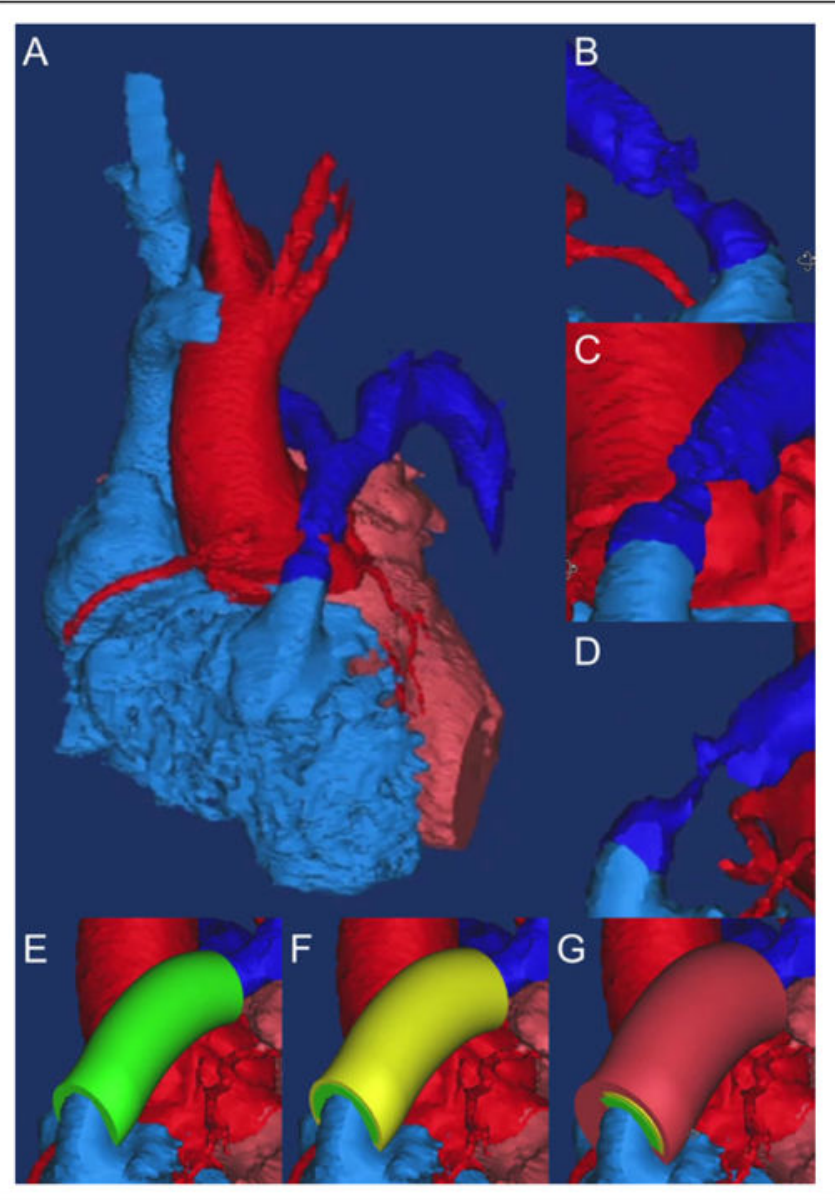

Figure 1.

Virtual right ventricle-to-pulmonary artery (RV-PA) conduit reconstruction surgery. A, Overview. B-D, Views of the RV-PA stenosis. E-G, Virtual surgery with three different sizes of conduits. Yellow: $18 \mathrm{~mm}$, green: $20 \mathrm{~mm}$, and red: $22 \mathrm{~mm}$. 


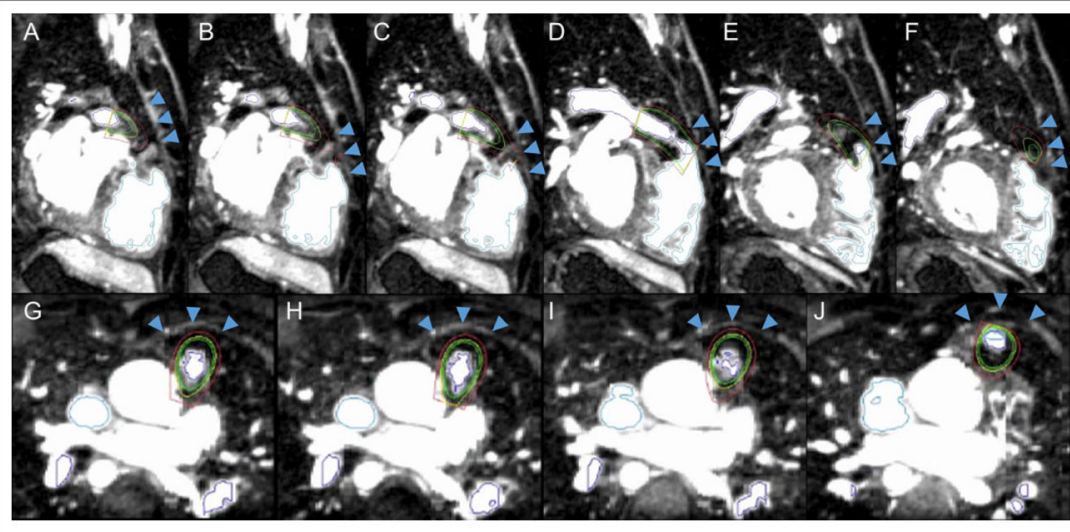

Figure 2.

Virtual simulated right ventricle-to-pulmonary artery (RV-PA) conduit superimposed on the patient's existing magnetic resonance imaging (MRI). These images are useful to estimate the relation of the virtual simulated conduit to the chest wall. A-F, Sagittal plane. G-J, Transverse plane. Blue arrowheads: inner chest wall; yellow: $18 \mathrm{~mm}$, green: $20 \mathrm{~mm}$, and red: $22 \mathrm{~mm}$. 